\title{
Expedient route to meroterpenoids
}

\author{
Nat. Chem. 12, 173-179 (2020)
}

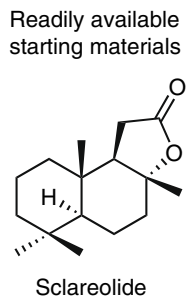

(1)

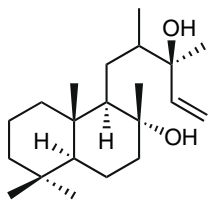

Sclareol

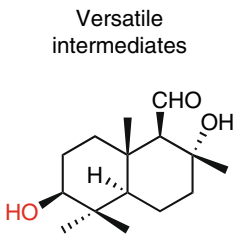

(3)

Biocatalytic oxidation HAT Giese coupling

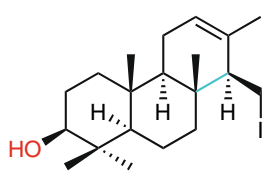

(4)
Meroterpenoid natural products<smiles>[Y6]C1CC2O[C@]3(C)CC[C@H]4C(C)(C)[C@@H](O)CC[C@]4(C)[C@H]3C([R7])C2C(=O)O1</smiles>

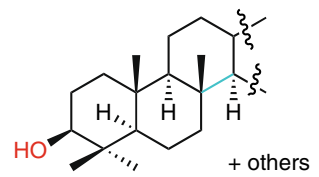

7-12 steps

Meroterpenoids are hybrid terpenoidpolyketide natural products showing therapeutic potential. Their complex and diverse structures provide a challenge for their chemical total synthesis and divergent strategies to produce these compounds are lacking.

Now, a team led by Hans Renata at The Scripps Research Institute used a retrosynthetic approach in order to design a short, versatile and efficient total synthesis of several members of this natural product family. The synthetic route employs sclareolide (1) and sclareol (2) as readily available starting materials, which are used as feedstocks in the perfume industry. Sclareolide (1) and sclareol (2) were converted into intermediates (3) and (4), in which selective hydroxylation at the C3 position constituted a major challenge for chemical synthesis. Such C3-selectivity is displayed in the enzyme universe by a cytochrome $\mathrm{P} 450$ variant, but its activity for the substrates here turned out to be rather poor. Using an alanine scan, the researchers were able to identify enzyme mutants with improved performance enabling suitable conversion. Further, after a hydrogen atom transfer (HAT)based Giese coupling, a third fused ring was generated leading to the construction intermediate (4). Using the intermediates (3) and (4) as platform compounds and employing the unique reactivity of radical-based transformations - largely avoiding the requirement of protection groups - allowed the straightforward synthesis of diverse meroterpenoids. In total eight meroterpenoid natural products were produced in just 7-12 steps from the commercially available starting materials (1) and (2). Notably, the step-count and yields compare favourably to previous reports and four natural products were accessed for the first time via chemical total synthesis.

This work exemplifies the value of versatile scientific training. Retrosynthesis elucidated an attractive pathway, for which realization was enabled by tools from bioand chemocatalysis; and molecular biology facilitated optimization of the enzyme to fit the chemist's requirements. Taking full advantage of the synthetic opportunities offered by different disciplines is expected to unlock more expedient routes to complex natural products and other valuable compounds.

\section{Jan-Stefan Völler}

Published online: 19 February 2020

https://doi.org/10.1038/s41929-020-0436-y 